\title{
Non-parametric shape optimization method for thin-walled structures under strength criterion
}

\author{
M. Shimoda ${ }^{1}$, J. Tsuji ${ }^{2}$ \& H. Azegami ${ }^{3}$ \\ ${ }^{1}$ Department of Mechanical Design Engineering, \\ Shonan Institute of Technology, Japan \\ ${ }^{2}$ Mitsubishi Automotive Engineering Co., Ltd., Japan \\ ${ }^{3}$ Nagoya University, Japan
}

\begin{abstract}
This paper presents a numerical optimization method for shape design to improve the strength of thin-walled structures. A solution to maximum stress minimization problems subject to a volume constraint is proposed. With this solution, the optimal shape is obtained without any parameterization of the design variables for shape definition. It is assumed that the design domain is varied in the in-plane direction to maintain the curvatures of the initial shape. The problem is formulated as a non-parametric shape optimization problem. The shape gradient function is theoretically derived using the Lagrange multiplier method and the adjoint variable method. The traction method, which was proposed as a gradient method in Hilbert space, is applied to determine the smooth domain variation that minimizes the objective functional. The calculated results show the effectiveness and practical utility of the proposed solution in solving minmax shape optimization problems for the design of thin-walled structures under a strength criterion.

Keywords: shell, shape optimization, traction method, structural optimization, optimal shape, non-parametric optimisation, minmax, adjoint variable.
\end{abstract}

\section{Introduction}

Thin-walled structures such as plates and shells are characterized by their ability to efficiently bear externally applied forces by means of the resultant membrane stress and bending stress. One can find many examples of such structures in the natural world, including leaves, seashells, eggshells and beetle shells, among 
others. In addition, such structures are also widely used as the basic framework of man-made artifacts such as automobile bodies, architectural structures and containers. While lightweight structures can be designed that take advantage of the features of thin-walled structures, assurance of the requirements concerning rigidity, vibration and strength is an essential design condition. Various methods can be used for reinforcing thin-walled structures such as by applying ribs, the overlapping of plates and shape modification. From the standpoint of design, the most desirable method is shape optimization because the application of ribs or the overlapping of structures is apt to result in weight increases or a decline in strength due to welding.

We previously proposed a shape optimization solution for the rigidity and natural vibration issues inherent in thin-walled structures [1,2]. This paper presents a solution to strength problems involving the minimization of maximum stress. The design variables that determine the shapes of thin-walled structures can vary in the in-plane direction and/or the out-of-plane direction. In this work, however, it was assumed that the domain boundaries varied in the in-plane direction so as to maintain the curvatures of the initial shape. This assumption is frequently encountered in the final design stage, and the method proposed here is especially effective at that stage of the design process.

In the case of minmax problems where maximum stress is the objective functional, a singularity issue occurs because the objective functional becomes a delta function. That issue was avoided here by transforming the local measure to an integral functional. The minmax problem considered was formulated as a non-parametric, or distributed parameter, shape optimization problem with a volume constraint. The sensitivity function, i.e., the shape gradient function, was theoretically derived using the Lagrange multiplier method, the material derivative method and the adjoint variable method. The optimal shape was then found using the traction method [3], a shape optimization method developed by the authors that does not require parameterization of the design variables. The effectiveness and practical utility of the proposed method were verified by applying it to basic examples of shape optimization problems and an actual automotive part.

\section{Variational equation for thin-walled structures}

Consider that a shell structure is a set of piecewise flat shell elements occupying a bounded domain $\Omega \subset \mathbb{R}^{3}$ as shown in Fig. 1 and eqn. (1) below. For simplicity, flat shell elements are used for discretizing the domain. The MindlinReissner plate theory is applied as the theory concerning plate bending, and coupling of the membrane stiffness and bending stiffness is ignored.

$$
\Omega=\left\{\left(x_{1}, x_{2}, x_{3}\right) \in \mathbb{R}^{3} \mid\left(x_{1}, x_{2}\right) \in A \subset \mathbb{R}^{2}, x_{3} \in(-h / 2, h / 2)\right\}
$$

where $S$ denotes the boundary of the design domain $A$ and $h$ is the plate thickness. Additionally, it is assumed that the mapping of the local coordinate 
system $\left(x_{1}, x_{2}, 0\right)$, which gives the position of the midsurface of the plate, to the global coordinate system $\left(X_{1}, X_{2}, X_{3}\right)$, i.e.,

$$
\phi:\left(x_{1}, x_{2}, 0\right) \in \mathbb{R}^{2} \mapsto\left(X_{1}, X_{2}, X_{3}\right) \in \mathbb{R}^{3}
$$

is piecewise smooth. The displacement expressed by the local coordinates, $\boldsymbol{u}=\left\{u_{i}\right\}_{i=1,2,3}$, is considered by dividing it into the displacement in the in-plane direction $\left\{u_{\alpha}\right\}_{\alpha=1,2}$, and the displacement in the out-of-plane direction $u_{3}$. The subscripts of the Greek letters are expressed as $\alpha=1,2$, and the tensor subscript notation with respect to $\alpha=1,2$ uses a summation convention and a partial differential notation $(\cdot)_{, i}=\partial(\cdot) / \partial x_{i}$ for the spatial coordinates. The MindlinReissner plate theory posits the following conditions with respect to displacement.

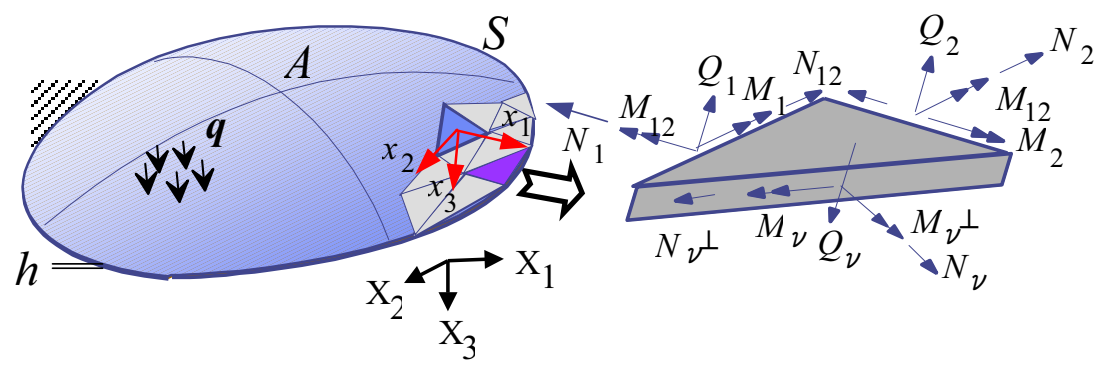

Figure 1: Geometry of shell and flat shell element.

$$
\begin{gathered}
u_{\alpha}\left(x_{1}, x_{2}, x_{3}\right) \equiv u_{0 \alpha}\left(x_{1}, x_{2}\right)-x_{3} \theta_{\alpha}\left(x_{1}, x_{2}\right) \\
u_{3}\left(x_{1}, x_{2}, x_{3}\right) \equiv w\left(x_{1}, x_{2}\right)
\end{gathered}
$$

where $\left\{u_{0 \alpha}\right\}_{\alpha=1,2}, w$ and $\left\{\theta_{\alpha}\right\}_{\alpha=1,2}$ express the in-plane displacement, out-ofplane displacement and rotational angle of the midsurface of the plate, respectively.

The boundary conditions shown in Fig. 1 for the shell are defined as follows. At the boundary $S$, the in-plane outward unit normal vector with respect to the boundary is expressed as $v=\left\{v_{\alpha}\right\}_{\alpha=1,2}$ relative to the local coordinate system $\left(x_{1}, x_{2}\right)$ and the unit tangent vector as $\boldsymbol{v}^{\perp}=\left\{v_{\alpha}^{\perp}\right\}_{\alpha=1,2} . \quad \boldsymbol{N}=\left\{N_{\alpha}\right\}_{\alpha=1,2}$, $\boldsymbol{M}=\left\{M_{\alpha}\right\}_{\alpha=1,2}$ and $\boldsymbol{Q}$ denote an in-plane force, a bending force and a shear force per unit length at the sub-boundaries in the boundary $S$, respectively. $\boldsymbol{q}$ is an out-of-plane on the domain $A$. Then, assuming the prescribed displacement and rotations are zero, the weak form of the equilibrium equation relative to $\left(\boldsymbol{u}_{0}, w, \theta\right) \in U$ can be expressed as

$$
a\left(\left(\boldsymbol{u}_{0}, w, \boldsymbol{\theta}\right),\left(\overline{\boldsymbol{u}}_{0}, \bar{w}, \overline{\boldsymbol{\theta}}\right)\right)=l\left(\left(\overline{\boldsymbol{u}}_{0}, \bar{w}, \overline{\boldsymbol{\theta}}\right)\right), \quad \forall\left(\overline{\boldsymbol{u}}_{0}, \bar{w}, \overline{\boldsymbol{\theta}}\right) \in U
$$


where $(\bullet)$ expresses a variation. $U$ denotes the function space of kinematically admissible displacements. In addition, the bilinear expression $a(\bullet, \bullet)$ and the linear expression $l(\bullet)$ are respectively defined as

$$
\begin{gathered}
a\left(\left(\boldsymbol{u}_{0}, w, \boldsymbol{\theta}\right),\left(\overline{\boldsymbol{u}}_{0}, \bar{w}, \overline{\boldsymbol{\theta}}\right)\right)=\int_{A}\left\{c_{\alpha \beta \gamma \delta}^{B} \theta_{(\gamma, \delta)} \bar{\theta}_{(\alpha, \beta)}\right. \\
\left.+c_{\alpha \beta \gamma \delta}^{M} u_{0(\gamma, \delta)} \bar{u}_{0(\alpha, \beta)}+k c_{\alpha \beta}^{S}\left(w_{\beta}-\theta_{\beta}\right)\left(\bar{w},_{\alpha}-\bar{\theta}_{\alpha}\right)\right\} d A \\
l\left(\left(\overline{\boldsymbol{u}}_{0}, \bar{w}, \overline{\boldsymbol{\theta}}\right)\right)=\int_{A} q \bar{w} d A+\int_{S}\left(N_{\alpha} \bar{u}_{0 \alpha}+M_{\alpha} \bar{\theta}_{\alpha}+Q \bar{w}\right) d S
\end{gathered}
$$

where $\left\{c_{\alpha \beta \gamma \delta}^{B}\right\}_{\alpha, \beta, \gamma, \delta=1,2},\left\{c_{\alpha \beta}^{S}\right\}_{\alpha, \beta=1,2}$ and $\left\{c_{\alpha \beta \gamma \delta}^{M}\right\}_{\alpha, \beta, \gamma, \delta=1,2}$ express an elastic tensor with respect to bending, shearing and membrane stress, respectively. The notation $k$ denotes a shear correction factor. In addition, $\left\{\kappa_{\alpha \beta}\right\}_{\alpha, \beta=1,2}$ and $\left\{\varepsilon_{\alpha \beta}\right\}_{\alpha, \beta=1,2}$ express the curvatures and the strains by $\left\{u_{0 \alpha \beta}\right\}_{\alpha, \beta=1,2}$ are defined by the following expressions. The total strains are expressed as eqns. (9) and (10).

$$
\begin{gathered}
\kappa_{\alpha \beta}=\frac{1}{2}\left(\theta_{\alpha, \beta}+\theta_{\beta, \alpha}\right) \equiv \theta_{(\alpha, \beta)}, \varepsilon_{0 \alpha \beta}=\frac{1}{2}\left(u_{0 \alpha, \beta}+u_{0 \beta, \alpha}\right) \equiv u_{0(\alpha, \beta)} \\
\varepsilon_{\alpha \beta}=u_{(\alpha, \beta)}=u_{0(\alpha, \beta)}-x_{3} \theta_{(\alpha, \beta)}, \quad \varepsilon_{\alpha 3}=u_{(\alpha, 3)}=\left(w_{, \alpha}-\theta_{\alpha}\right) / 2
\end{gathered}
$$

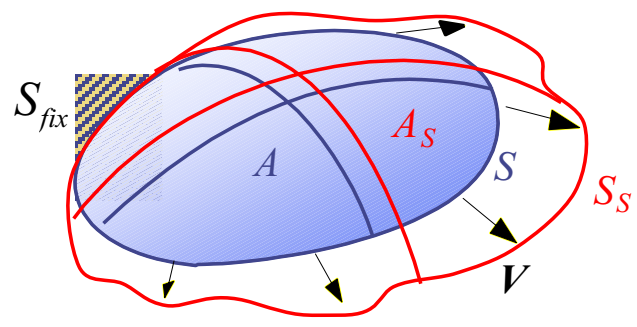

Figure 2: $\quad$ In-plane domain variation $\boldsymbol{V}$.

\section{Formulation and solution of minmax problem under strength criterion}

As shown in Fig. 2, consider that a linear shell having an initial domain $A$ and boundary $S$ undergoes in-plane domain variation (i.e., the design velocity field) $\boldsymbol{V}$ such that its domain and boundary become $A_{s}$ and $S_{s}$. The domain variation can be expressed by a one-parameter family $T_{S}: \mathbb{R}^{2} \mapsto \mathbb{R}^{2}, 0 \leq s \leq \varepsilon$ ( $\varepsilon$ is a small integer) of the mapping from $\left(x_{1}, x_{2}\right) \in A$ to $\left(x_{s 1}, x_{s 2}\right) \in A_{s}$. The notation $s$ indicates the iteration history of domain variation. 
The non-parametric shape optimization problem with the objective of minimizing the maximum value of von Mises stress $\sigma_{M}$ on both sides of a shell structure can be formulated as shown below, subject to constraints of volume and the state equation in eqn. (4). It is assumed that the plate thickness does not vary.

Find $A$

that minimize $\max _{x \in A} \max _{m=\text { top }, \text { bottom }} \frac{\sigma_{M}^{(m)}(x)}{\sigma_{a}}$

subject to Eq.(4),

$$
M\left(=\int_{A} h d A\right) \leq \hat{M} .
$$

where $M$ and $\hat{M}$ are the volume and its constraint value and $\sigma_{a}$ is a constant for the purpose of normalization. $\sigma_{M}$ is defined as eqn. (15), and calculated by using eqns. (9)(10) and the Lame coefficients.

$$
\sigma_{M}\left(\sigma_{i j}\left(\boldsymbol{u}_{0}(x), w(x), \theta(x)\right)\right)=\left\{\sigma_{11}^{2}+\sigma_{22}^{2}-\sigma_{11} \sigma_{22}+3\left(\sigma_{12}^{2}+\sigma_{13}^{2}+\sigma_{23}^{2}\right)\right\}^{\frac{1}{2}}
$$

The issues of non-differentiability are inherent to stress minmax problems because of the singularity of maximum stress, making it theoretically difficult to determine directly the sensitivity of the local objective functional in eqn. (12). Therefore, the Kreisselmeier-Steinhauser $(K S)$ function [4] is used to transform the local objective functional into the following smooth differentiable integral functional. When a constant $\rho$ is sufficiently large, the maximum value can be extracted. In actuality, a value of $\rho$ in a range of 5 to 200 is used.

$$
\max _{x \in A} \max _{m=\text { top }, \text { botom }} \frac{\sigma_{M}^{(m)}(x)}{\sigma_{a}} \Rightarrow \frac{1}{\rho} \ln \int_{A}\left\{\sum_{m=\text { top }}^{\text {bottom }} \exp \left(\frac{\sigma_{M}^{(m)}}{\sigma_{a}} \rho\right)\right\} d A
$$

Letting $\left(\overline{\boldsymbol{u}}_{0}, \bar{w}, \overline{\boldsymbol{\theta}}\right)$ and $\Lambda$ denote the Lagrange multipliers of the state equation and volume constraint, respectively, the Lagrangian functional $L$ for this problem can be expressed as

$$
\begin{aligned}
& L\left(\boldsymbol{u}_{0}, w, \boldsymbol{\theta}, \overline{\boldsymbol{u}}_{0}, \bar{w}, \overline{\boldsymbol{\theta}}, \Lambda\right)=\frac{1}{\rho} \ln \int_{A}\left\{\sum_{m} \exp \left(\frac{\sigma_{M}^{(m)}}{\sigma_{a}} \rho\right)\right\} d A \\
& +l\left(\left(\overline{\boldsymbol{u}}_{0}, \bar{w}, \overline{\boldsymbol{\theta}}\right)\right)-a\left(\left(\boldsymbol{u}_{0}, w, \boldsymbol{\theta}\right),\left(\overline{\boldsymbol{u}}_{0}, \bar{w}, \overline{\boldsymbol{\theta}}\right)\right)+\Lambda(M-\hat{M})
\end{aligned}
$$

For simplicity, it is assumed that the boundary on which the load acts does not vary in the normal direction and that the domain on which the out-of-plane force $q$ acts does not vary. Then, the material derivative $\dot{L}$ with respect to the domain variation of the Lagrangian functional can be expressed as

$$
\begin{gathered}
\dot{L}=\frac{1}{\sigma_{a} B} \int_{A} \sum_{m} \exp \left(\frac{\sigma_{M}^{(m)}}{\sigma_{a}} \rho\right) \frac{\partial \sigma_{M}}{\partial \sigma_{i j}} \sigma_{i j}^{\prime} d A-a\left(\left(\boldsymbol{u}_{0}^{\prime}, w^{\prime}, \boldsymbol{\theta}^{\prime}\right),\left(\overline{\boldsymbol{u}}_{0}, \overline{\boldsymbol{w}}, \overline{\boldsymbol{\theta}}\right)\right)+l\left(\left(\overline{\boldsymbol{u}}_{0}^{\prime}, \bar{w}^{\prime}, \overline{\boldsymbol{\theta}}^{\prime}\right)\right) \\
-a\left(\left(\boldsymbol{u}_{0}, w, \boldsymbol{\theta}\right),\left(\overline{\boldsymbol{u}}_{0}^{\prime}, \overline{\boldsymbol{w}}^{\prime}, \overline{\boldsymbol{\theta}}^{\prime}\right)\right)+\Lambda^{\prime}(M-\hat{M})+\int_{S} G V_{v} d s, \quad \boldsymbol{V} \in C_{\Theta} \\
B=\int_{A} \sum_{m} \exp \left(\left(\sigma_{M}^{(m)} / \sigma_{a}\right) \rho\right) d A
\end{gathered}
$$


184 Computer Aided Optimum Design in Engineering X

$$
\begin{aligned}
G=-c_{\alpha \beta \gamma \delta}^{B} \theta_{(\gamma, \delta)} \bar{\theta}_{(\alpha, \beta)}- & c_{\alpha \beta \gamma \delta}^{M} u_{0(\gamma, \delta)} \bar{u}_{0(\alpha, \beta)}-k c_{\alpha \beta}^{S}\left(w_{, \beta}-\theta_{\beta}\right)\left(\bar{w}_{, \alpha}-\bar{\theta}_{\alpha}\right) \\
& +\frac{1}{\rho B} \sum_{m} \exp \left(\frac{\sigma_{M}^{(m)}}{\sigma_{a}} \rho\right)+\Lambda
\end{aligned}
$$

where $\boldsymbol{V}(x)=\partial T_{s} / \partial s\left(T_{s}^{-1}(x)\right), x \in A_{s}$ is the velocity field in relation to the domain variation $s . \quad V_{v}=v_{i} V_{i}$. The notation ( $)^{\prime}$ is the shape derivative and $\left.{ }^{\circ}\right)$ is the material derivative [5]. $C_{\Theta}$ is the suitably smooth function space that satisfies the constraints of domain variation. $G$ is called the shape gradient density function.

The optimality conditions of the Lagrangian functional $L$ with respect to $\left(\boldsymbol{u}_{0}, w, \theta\right),\left(\overline{\boldsymbol{u}}_{0}, \bar{w}, \bar{\theta}\right)$ and $\Lambda$ are expressed as shown below.

$$
\begin{gathered}
a\left(\left(\boldsymbol{u}_{0}, w, \boldsymbol{\theta}\right),\left(\overline{\boldsymbol{u}}_{0}^{\prime}, \bar{w}^{\prime}, \overline{\boldsymbol{\theta}}^{\prime}\right)\right)=l\left(\left(\overline{\boldsymbol{u}}_{0}^{\prime}, \bar{w}^{\prime}, \overline{\boldsymbol{\theta}}^{\prime}\right)\right), \forall\left(\overline{\boldsymbol{u}}_{0}^{\prime}, \bar{w}^{\prime}, \overline{\boldsymbol{\theta}}^{\prime}\right) \in U \\
a\left(\left(\boldsymbol{u}_{0}^{\prime}, w^{\prime}, \boldsymbol{\theta}^{\prime}\right),\left(\overline{\boldsymbol{u}_{0}}, \bar{w}, \overline{\boldsymbol{\theta}}\right)\right)=\frac{1}{\sigma_{a} B} \int_{A} \sum_{m} \exp \left(\frac{\sigma_{M}^{(m)}}{\sigma_{a}} \rho\right) \frac{\partial \sigma_{M}}{\partial \sigma_{i j}} \sigma_{i j}^{\prime} d A, \\
\forall\left(\boldsymbol{u}_{0}^{\prime}, w^{\prime}, \boldsymbol{\theta}^{\prime}\right) \in U \\
\Lambda(M-\hat{M})=0 \\
M-\hat{M}=0 \\
\Lambda \geq 0
\end{gathered}
$$

By substituting $\left(\boldsymbol{u}_{0}, w, \boldsymbol{\theta}\right),\left(\overline{\boldsymbol{u}}_{0}, \bar{w}, \overline{\boldsymbol{\theta}}\right)$ and $\Lambda$ determined by the state equation (eqn. (21)), the adjoint equation (eqn. (22)) and eqns. (23)-(25) into eqn. (18), the material derivative $\dot{L}$ can be expressed as the dot product of the shape gradient function $\boldsymbol{G}$ and the design velocity field $\boldsymbol{V}$ as shown in eqn. (26). Considering $\sigma_{33}=0$, the adjoint equation can be solved by applying the distributed initial strain tensor $\left\{\frac{1}{\sigma_{a} B} \sum_{m} \exp \left(\frac{\sigma_{M}^{(m)}}{\sigma_{a}} \rho\right) \frac{\partial \sigma_{M}}{\partial \sigma_{i j}}\right\}$ in the domain as an external force.

$$
\dot{L}=l_{G}(\boldsymbol{V}) \equiv \int_{S_{s}} G_{i} V_{i} d \Gamma
$$

The shape gradient function $\boldsymbol{G}$ can be expressed as shown below.

$$
\begin{gathered}
\boldsymbol{G}=\left[-c_{\alpha \beta \gamma \delta}^{B} \theta_{(\gamma, \delta)} \bar{\theta}_{(\alpha, \beta)}-c_{\alpha \beta \gamma \delta}^{M} u_{0(\gamma, \delta)} \bar{u}_{0(\alpha, \beta)}-k c_{\alpha \beta}^{S}\left(w_{, \beta}-\theta_{\beta}\right)\left(\bar{w}_{, \alpha}-\bar{\theta}_{\alpha}\right)\right. \\
\left.+\frac{1}{\rho B} \sum_{m} \exp \left(\frac{\sigma_{M}^{(m)}}{\sigma_{a}} \rho\right)+\Lambda\right] v
\end{gathered}
$$

Since the shape gradient function has been derived, the traction method can be applied.

\section{Traction method}

The traction method is a gradient method in Hilbert space [3]. With the traction method, the domain variation (i.e., design velocity field $\boldsymbol{V}$ ) is found as a 
displacement field when a negative shape gradient function $-\boldsymbol{G}$ acts as a distributed traction force on the design boundaries that allow domain variation under a shape constraint condition in a pseudo-elastic problem. The governing equation for applying the traction method to thin-walled structures considered here is given as the expression noted below. Equation (28) can be solved by a standard finite element analysis. We call this calculation the velocity analysis.

$$
a\left(\boldsymbol{V}, \boldsymbol{y}_{0}\right)=-l_{G}\left(\boldsymbol{y}_{0}\right), \quad \forall \boldsymbol{y}_{0} \in C_{\Theta}
$$

In order to maintain the curvatures of the initial shape, we constrain the variation in the normal direction to the shell surface. By repeating the stress, the velocity analysis and the updating of the shape, the objective functional is minimized, resulting in the optimized shape.

\section{Results of numerical calculations}

To confirm the validity of the proposed method, it was applied to two simple design problems and to an actual automotive part.

\subsection{Shell with hole}

The shape of a round hole in the center of a shell was optimized. One end of the shell was clamped and a downward distributed load was applied to the other end. The boundary conditions of the stress analysis and of the velocity analysis are shown in Fig. 3-(a) and (b), respectively. A constant volume constraint was applied. The initial shape and the optimized shape are shown respectively in Fig. 4-(a) and (b). It is seen that the round hole changed to a smooth optimal shape while maintaining the initial curvature of the shell. Figure 5 shows iteration histories of the volume and objective functional (eqn. (16)) in the optimization process and of the maximum von Mises stress. The values have been normalized to the values of the initial shape. It is clear from the results that the objective functional and the maximum von Mises stress were minimized while satisfying the volume constraint. The objective functional was reduced by $8 \%$ and the maximum stress by $9 \%$.

\subsection{Plate with holes}

The shape of a cantilever plate with three round holes was optimized as shown in Fig. 6. One end of the plate was clamped and a perpendicular load was applied to the boundary at the other end. In Case 1, design boundaries were defined at the two outer sides of the plate and the shape of the round holes was not changed. In Case 2, the boundaries of the three holes were set as the design boundaries and the outer shape of the plate was not changed. A constant volume was applied as the constraint value. The optimized shapes obtained in Case 1 and Case 2 are shown in Fig. 7-(a) and (b), respectively. It is seen that a smooth converged shape was obtained in each case. In both cases, the objective functional 
decreased monotonically while satisfying the constraint condition. The maximum stress was reduced by approximately $53 \%$ in Case 1 and by approximately $55 \%$ in Case 2 .

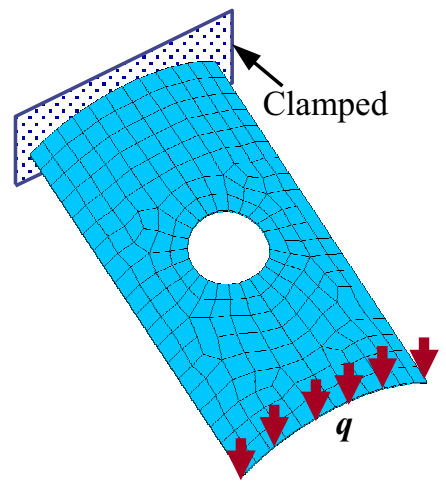

(a) Stress Analysis

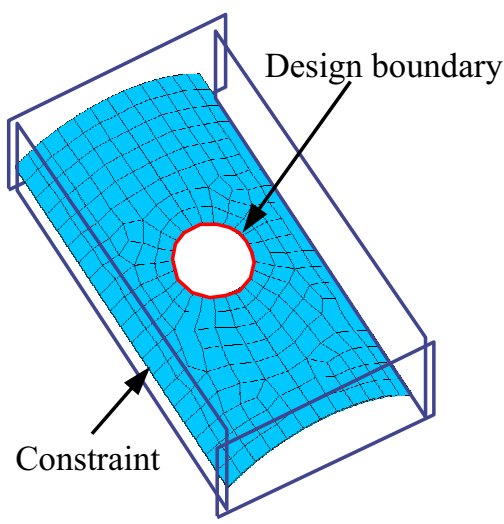

(b) Velocity Analysis

Figure 3: Boundary conditions for shape optimization of shell with hole.
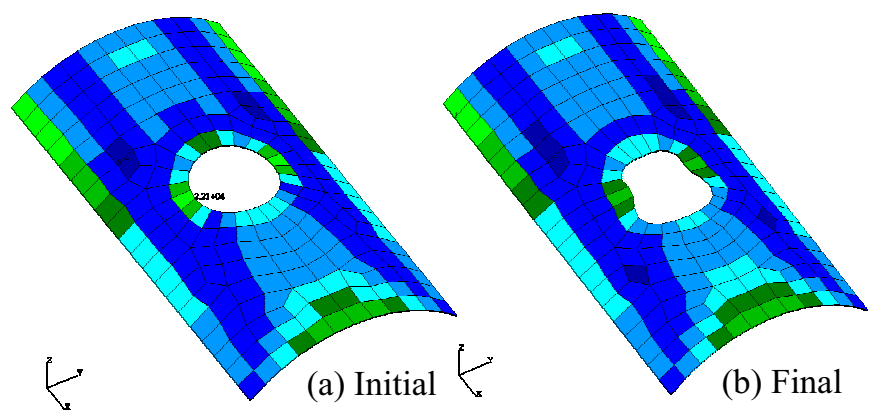

Figure 4: $\quad$ Calculated results for shell with hole.

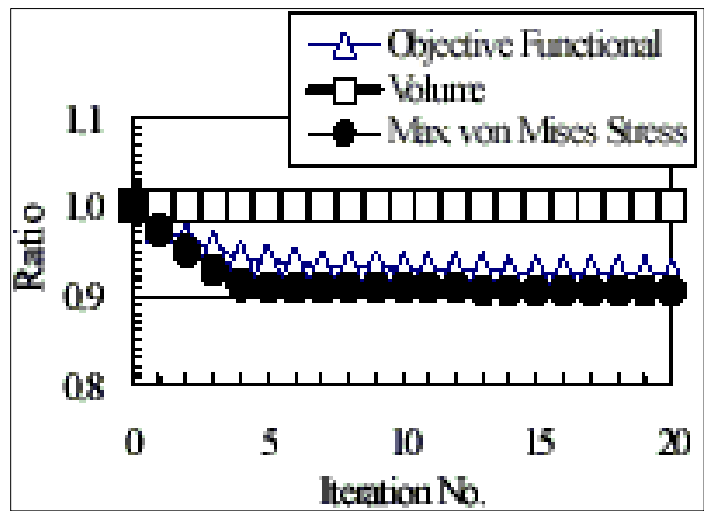

Figure 5: Iteration histories of shell with hole. 


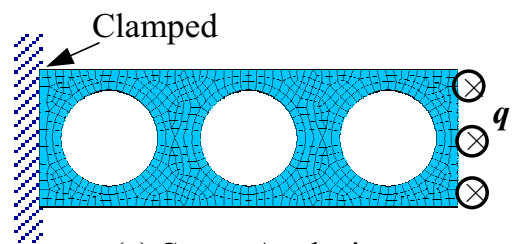

(a) Stress Analysis

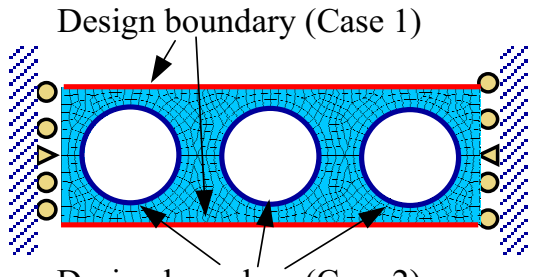

Design boundary (Case 2)

(b) Velocity Analysis

Figure 6: Boundary conditions for optimization of plate with holes.

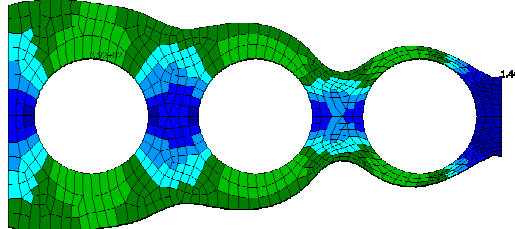

(a) Case 1 (Edge design)

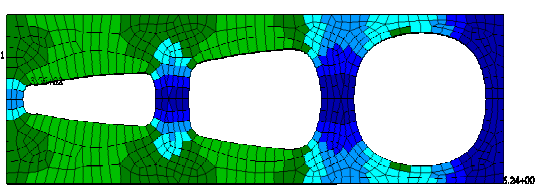

(b) Case 2 (Hole design)

Figure 7: Calculated results for plate with holes.

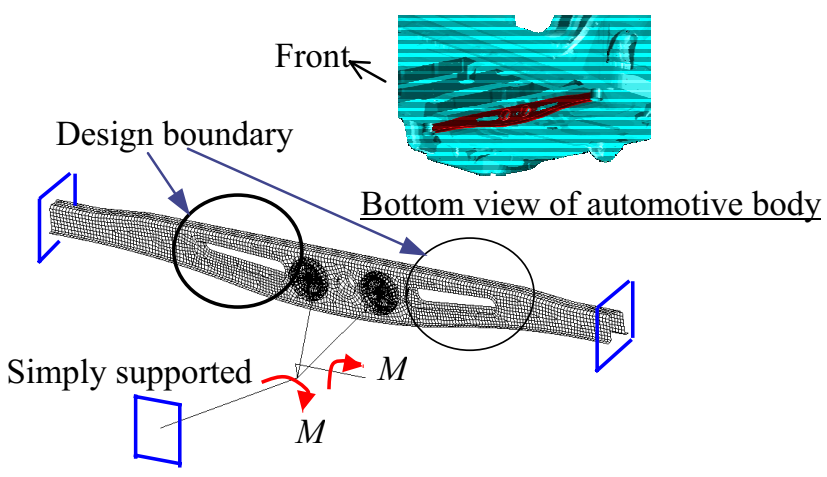

Figure 8: Boundary condition for shape optimization of suspension part.

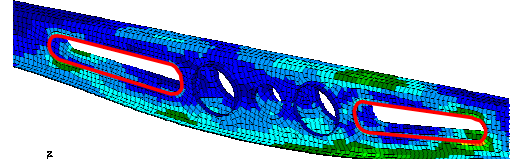

(a) Initial

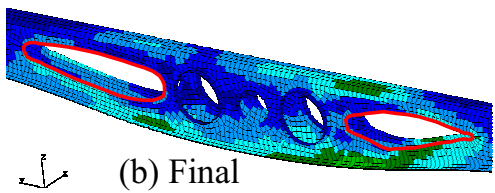

(b) Final

Figure 9: Calculated results for suspension part. 


\subsection{Application to a suspension part}

The proposed method was also applied to an actual automotive suspension part to verify its validity. Figure 8 shows the initial shape of the suspension part used and the design domain (hole shape). Figure 9-(a) shows the initial part shape and Fig. 9-(b) shows the optimized shape obtained. A constant volume constraint was also applied in this case. The maximum stress was reduced by approximately $43 \%$ while satisfying the constraint condition.

\section{Conclusion}

This paper has described a solution to shape optimization problems of thinwalled structures subject to a strength criterion. The shape optimization method proposed here minimizes the maximum von Mises stress under a volume constraint while assuming that the curvatures of the initial shape are maintained. With this approach, the shape gradient function of the problem is theoretically derived, and the traction method is then applied to determine the smooth optimal shape. It can be easily implemented without any parameterization of the design variables for shape definition. As a result of analyzing the optimal shapes of simple design problems and an actual automotive part, it was confirmed that the method functioned as intended and produced good shape optimization results, the method will especially effective at the final stage of the design process.

\section{References}

[1] Shimoda M., Tsuji J. and Azegami H., Minimum Weight Shape Design for the Natural Vibration Problem of Plate and shell structures, Transactions of the Wessex Institute on the Built Environment, 80, (Computer Aided Optimum Design of Structures IX), S. Hernandez and C. A. Brebbia (eds.), WIT Press, Southampton, pp. 147-156, 2005.

[2] Shimoda M. and Tsuji J., Non-parametric Shape Optimization Method for Rigidity Design of Automotive Sheet Metal Structures, Proceedings of SAE World Congress 2006, SAE Paper 200601-0584, 2006.

[3] Azegami H. and Wu Z. C., Domain Optimization Analysis in Linear Elastic Problems (Approach Using Traction Method), JSME Int. J., Series A, 39(2), pp. 272-278, 1996.

[4] Kreisselmeier G. and Steinhauser R., Systematic Control Design by Optimizing a Vector Performance Index, Int. Federation of Active Control Symposium on Computer-Aided Design of Control Systems, pp.113-117, 1979.

[5] Sokolowski, J. and Zolesio, J. P., Introduction to Shape Optimization Shape Sensitivity Analysis, Springer-Verlag: New York, 1991. 